\title{
Influence of Confining Pressure on the Crack Growth Behavior in a Highly Filled Elastomer
}

Materials Technology Center, Southern Illinois University,

Carbondale, IL 62901

\section{G. Ravichandran}

Graduate Aeronautical Laboratories, California Institute of Technology, Pasadena, CA 91125
In this study, the effect of confining pressure on the crack growth behavior in a highly filled elastomer was investigated. The material under investigation contains $86 \%$ by weight of hard particles embedded in a rubbery matrix. The specimens were tested at a constant strain rate under a confining pressure. Two confining pressures, $3.45 \mathrm{MPa}$ and $6.9 \mathrm{MPa}$, and two types of loading, monotonically increasing and cyclic, were considered. The experimental data were analyzed, and the effects of confining pressure on the damage process as well as the constitutive and crack growth behavior in the material are discussed. [DOI: 10.1115/1.2189874]

\section{Introduction}

An important engineering problem in structural design is evaluating its integrity and reliability. It is well known that structural strength may be degraded during its design life due to mechanical or chemical aging, or a combination of these two aging mechanisms. Depending on the structural design, material type, service loading, and environmental condition, the cause and degree of strength degradation due to the different aging mechanisms differs. One of the common causes of strength degradation is the result of damage and crack development in the structure. Therefore, to effectively use the material in structural applications one needs to understand the damage initiation and evolution processes, the effects of damage and crack development on the material's response, and the remaining strength and life of the structures.

In recent years, a considerable amount of work has been done studying crack growth behavior in particulate composite materials under different loading conditions at ambient pressure [1-4]. Experimental findings indicate that power-law relationships exist between the crack growth rate, $d a / d t$, and the mode I stress intensity factor $K_{\mathrm{I}}$. These experimental findings support the theory developed by Knauss [5] and Schapery [6] in their studies of crack growth behavior in linear viscoelastic materials. It is known that classical fracture mechanics principles, especially linear elastic fracture mechanics, are well established for single-phase materials. Experimental data indicate that linear fracture mechanics theories can be applied to the particulate composite materials with varying degrees of success. However, there has been relatively little effort in understanding the crack growth behavior in such materials under confining pressure condition.

In this study, uniaxial specimens with and without precrack were used to investigate the effects of confining pressure on the constitutive and crack growth behavior in a highly filled elastomer. The material contains $86 \%$ by weight of hard particles embedded in a rubbery matrix. The specimens were tested under confining pressure $3.45 \mathrm{MPa}$ and 6.9 MPa. The specimens without precrack were tested at $16.6 \mathrm{~cm} / \mathrm{cm} / \mathrm{min}$ and $0.073 \mathrm{~cm} / \mathrm{cm} / \mathrm{min}$ for monotonically increasing loading and cyclic loading, respectively. The specimens with precrack were

Contributed by the Applied Mechanics Division of ASME for publication in the Journal of Applied Mechanics. Manuscript received January 18, 2005; final manuscript received January 20, 2006. Review conducted by K. Ravi-Chandar. Discussion on the paper should be addressed to the Editor, Prof. Robert M. McMeeking, Journal of Applied Mechanics, Department of Mechanical and Environmental Engineering, University of California-Santa Barbara, Santa Barbara, CA 93106-5070, and will be accepted until four months after final publication of the paper itself in the ASME JoURNAL OF APPLIED MECHANICS. tested under monotonically increasing loading at a strain rate of $16.7 \mathrm{~cm} / \mathrm{cm} / \mathrm{min}$ only. The experimental data were analyzed, and the effects of confining pressure on the damage process as well as the constitutive and crack growth behavior in the material are discussed.

\section{Experiments}

In this study, a series of experiments were conducted on uniaxial specimens with and without precrack under a confining pressure. Two different confining pressures, $3.45 \mathrm{MPa}$ and 6.9 $\mathrm{MPa}$, were considered. The specimens were made of a highly filled polymeric material, containing $86 \%$ by weight of hard particles embedded in a rubbery matrix. The dimensions of the specimen were $7.6 \mathrm{~cm}$ long, $2.54 \mathrm{~cm}$ wide, and $5.08 \mathrm{~cm}$ thick (Fig. 1). For the precracked specimens, a $0.762 \mathrm{~cm}$ crack was cut in the middle at one of the long edges of the specimen with a razor blade. Prior to conducting the tests, the specimen was loaded in a materials testing machine inside a pressure chamber. When the pressure inside the pressure chamber reached the predetermined confining pressure, the specimen was deformed at a constant strain rate of $16.7 \mathrm{~cm} / \mathrm{cm} / \mathrm{min}$ until the specimen fractured. In addition, two cyclic loading tests were also conducted to investigate the effect of confining pressure on the damage state and the constitutive behavior of the material. For the cyclic loading tests, the specimen was strained to $10 \%$ strain level, and then it was unloaded to zero stress. After waiting for $15 \mathrm{~min}$ at zero stress, the specimen was reloaded until fractured. For the cyclic loading tests, the specimens were deformed at a constant strain rate of $0.073 \mathrm{~cm} / \mathrm{cm} / \mathrm{min}$. For one of the two cyclic loading tests, the specimen was tested under $6.9 \mathrm{MPa}$ confining pressure, whereas, for the other one, the specimen was loaded and unloaded under 6.9 MPa confining pressure but reloaded under ambient pressure. During the deformation of the specimen, a high-speed camera was used to monitor the test. In addition, the load and time were also recorded. The raw data were used to determine the stress, the strain, mode I stress intensity factor, and crack growth rate.

To determine the stress intensity factor at the crack tip, it is necessary to relate the load on the specimen to crack and specimen dimension. A three-dimensional finite element computer code TEXGAP-3D was used to determine the stress intensity as a function of the crack lengths for a given load $P$ applied at the boundary of the specimen. The calculated mode I stress intensity factor $K_{\mathrm{I}}$ was normalized with respect to the applied load $P$. A linear regression analysis was conducted to determine the relationship between the normalized mode I stress intensity factor $K_{\mathrm{I}} / P$ to the crack length a. This relationship can be used to determine the mode I stress intensity factor for elastic and viscoelastic materials. In calculat- 


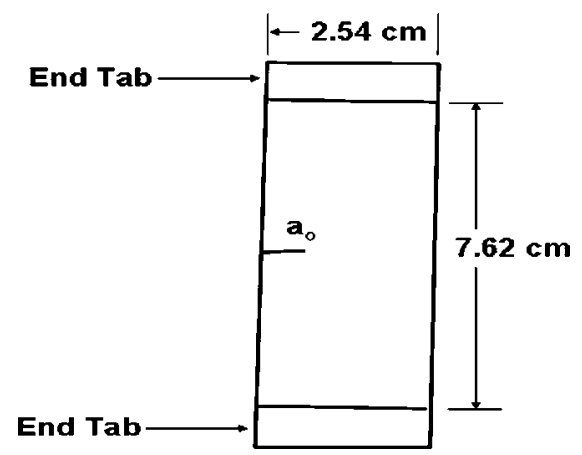

Fig. 1 Specimen geometry

ing $K_{\mathrm{I}}$ from experimental data, the recorded data, $a$ and $p$ for a given time, and the regression equation relating $K_{\mathrm{I}} / P$ and $a$ were used.

To determine the crack growth rate $d a / d t$, the polynomial method was used. This method involved fitting an $n$th order polynomial to a set of crack length versus time data. The coefficients of the polynomial function were estimated by the method of least squares. The crack growth rate was calculated by taking derivative of the polynomial function at a given time.

\section{Results and Discussion}

It is well known that, on the microscopic scale, a highly filled polymeric material can be considered to be an inhomogeneous material. When these materials are stretched, the different sizes and distribution of filled particles, the different cross-linking density of polymeric chains, and the variation in bond strength between the particles and the binder can produce highly inhomogeneous local stress and strength fields. Also, the material may contain randomly distributed microvoids, incipient damage sites, and microcracks with statistically distributed size and orientation. Therefore, the local strength in the material varies in a random fashion, so the failure sites in the material do not necessarily coincide with the maximum stress location. Hence, the failure location as well as the degree of damage induced in the material will also vary in a random fashion. Depending on the magnitude of the local stress and the local strength, damage can be developed in the material, especially near the crack tip region. The damage developed in the material may be in the form of microvoids or microcracks in the binder or dewetting between the binder and the filler particles. When the particle is dewetted, the local stress will be redistributed. With time, additional binder/particle separation and vacuole formation take place. The damage growth in the material may take place as material tearing or as successive nucleation and coalescence of the microcracks. These damage initiation and evolution processes are time dependent and are the main factor responsible for the time-dependent constitutive and fracture behavior of the material. It should be pointed that the aforementioned damage initiation and evolution processes are commonly observed in the particulate composite material studied when the material is subjected to a monotonically increasing load under low confining pressure. However, when the confining pressure is very high, the damage mechanisms may change, which is a subject of study. Having discussed the damage mechanisms in the particulate composite material, one can now discuss how the damage state affects the constitutive behavior of the material.

A typical plot of stress-strain curves, obtained from tests on specimens without precrack at a constant strain rate of $16.7 \mathrm{~cm} / \mathrm{cm} / \mathrm{min}$ under ambient, $3.45 \mathrm{MPa}$, and $6.90 \mathrm{MPa}$ confining pressures, are shown in Fig. 2. From Fig. 2, it is seen that the maximum load increase significantly when the pressure is increased from ambient to $6.90 \mathrm{MPa}$. However, the magnitude of the confining pressure has no significant effect on effective modu-

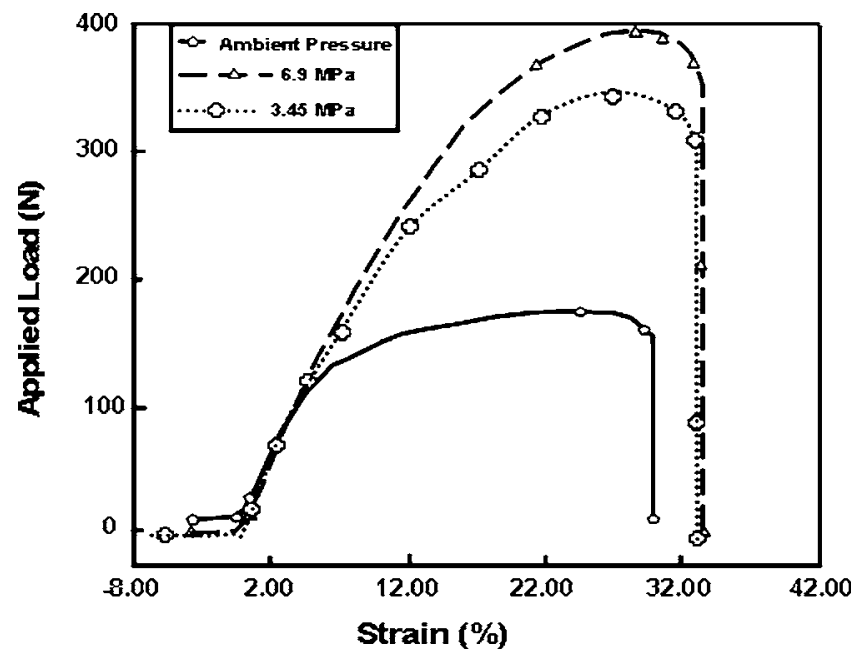

Fig. 2 Applied load versus applied strain under different confining pressure

lus. Theoretically, the magnitude of the confining pressure should have no effect on the Young's modulus due to the incompressibility of the material. The slight variation of the Young's modulus is due to the scatter of the test data, material variability, and the reason that the material is not truly incompressible. It is interesting to note that under the high confining pressure condition, the critical strain $\varepsilon_{\mathrm{c}}$ for the transition of the linear response to the nonlinear response of the material is increased. Since the material's response is closely related to the damage state in the material, the change of the aforementioned parameters is believed due to the suppression, or the delay, of the development of damage under the high confining pressure.

Throughout the loading history, the progressive development and interaction of various damage modes change the state of the material or the mechanical response of the material. In general, when the highly filled elastomer is deformed under constant strain-rate condition, the initial linear portion of the stress-strain curve is associated with stretching undamaged material, when the filler particles are bonded to the binder. As the external load is continuously increased, at a certain critical stress/strain level, dewetting occurs. When the density of the dewetted particles reaches a critical value, the rigidity of the material is thereby reduced, and usually this critical dewetting state coincides with the transition from linear response to nonlinear behavior. As the specimen is continuously stretched, the number of dewetted particles is increased and the voids that are formed begin to grow and coalesce. This damage process is related, primarily, to the nonlinear response of the material and can be characterized by bulk volume change during stretching. The bulk volume change during straining is usually known as the volume dilatation, which is partially caused by the nucleation of new voids and partially caused by the growth of the existing voids. The extent of the volume dilatation depends on the nature of the binder/particle system, the testing temperature, and the strain rate. The volume dilatation is used as a damage parameter and has been incorporated into a constitutive model [7], which, in turn, has been implemented in a deformation-based finite element simulation. The critical volume dilatation is used as a failure criterion in this phenomenological model for predicting the crack growth behavior in filled elastomers [8].

A typical plot of volume dilatation, measured by dilatometer, versus applied strain under different confining pressures is shown in Fig. 3. It is noted that for a given applied strain, the volume dilatation decreases as the confining pressure is increased. At 6.9 $\mathrm{MPa}$ confini ng pressure, the dilatation is very small. In order to see whether or not damage can develop in the material under 


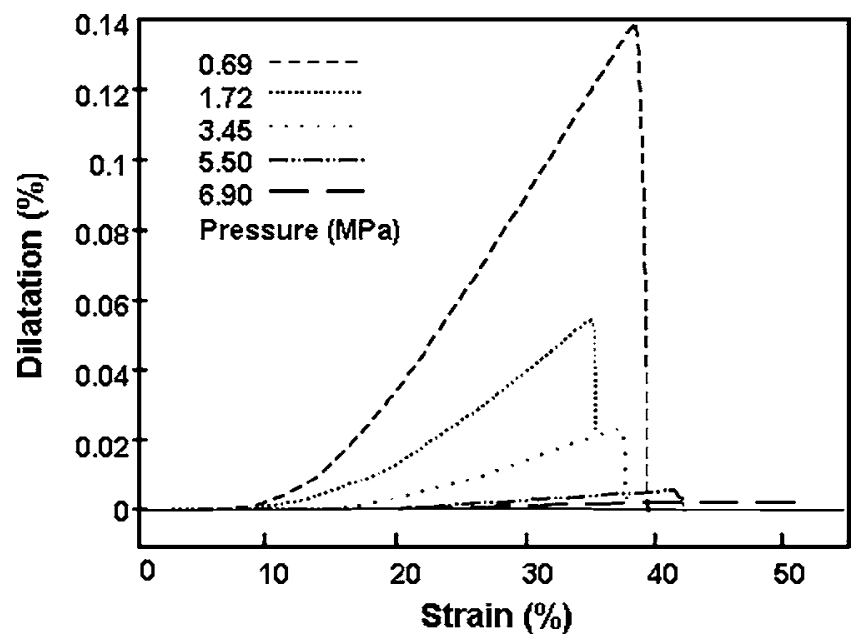

Fig. 3 Volume dilatation versus applied strain under different confining pressure

6.9 MPa confining pressure, cyclic loading experiments were conducted under $6.9 \mathrm{MPa}$ confining pressure, and the results are shown in Figs. 4 and 5. In addition to plotting the cyclic stressstrain curve, the stress-strain curve under a monotonic increasing loading condition with the same strain rate as the cyclic loading test is also plotted. From Fig. 4, it is seen that the slope of the initial part of the reloading curve is smaller than that of the loading curve corresponding to that of the cyclic loading. In other wards, after the cyclic loading, the modulus of the material is decreased, implying that, under 6.9 MPa confining pressure, the material can be damaged by the cyclic load. It is interesting to note that the reloading stress-strain curve rejoins the constant strain rate stress-strain curve, indicating that the cyclic loading considered in this study has no significant effect on the tensile strength of the material. The development of damage under confining pressure is further illustrated in Fig. 5, which shows the loading and unloading stress-strain curves under 6.9 MPa confining pressure and the reloading stress-strain curve under ambient pressure. It is noted that (similar to Fig. 4) the modulus of the material, after the specimen is subjected to the cyclic load under 6.9 $\mathrm{MPa}$ confining pressure, is decreased. However, the maximum stress is lower than that under the previous cyclic load. Based on the experimental findings, it is conjectured that under confining pressure, microcracks are developed near the particle surface where high triaxial tensile stresses are developed due to the high constraint developed near the particle surface. Under the high confining pressure condition, the microcracks cannot open up to form voids, resulting in very small volume dilatation. When the speci-

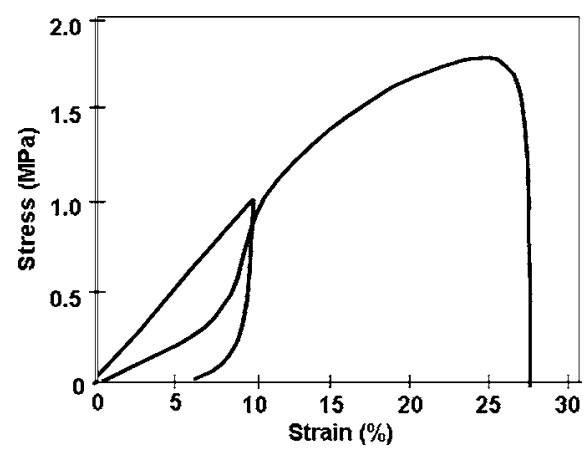

Fig. 4 Stress-strain curves under cyclic loading (loading, unloading, and reloading under $6.90 \mathrm{MPa}$ confining pressure)

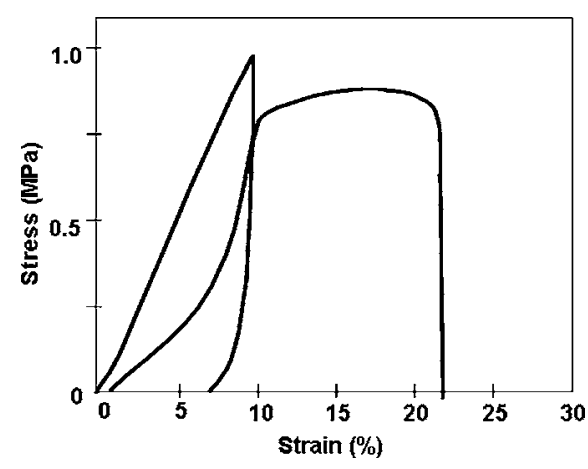

Fig. 5 Stress-strain curves under cyclic loading (loading and unloading under $6.90 \mathrm{MPa}$ confining pressure, reloading under ambient pressure)

men is reloaded under ambient pressure, the microcracks open up and grow. The growth of the microcracks results in the fracture of the specimen and a lower maximum stress.

The above paragraphs discussed the effect of confining pressure on the constitutive behavior and damage mechanisms in the highly filled elastomer. In the following paragraphs, we will discuss the effect of confining pressure on the crack growth behavior in the material

Experimental results indicate that crack tip blunting takes place both before and after crack growth. The material at the tip of the crack undergoes very large elongation, and the crack tip is nearly straight, indicating extensive blunting. The highly strained or damage zones extends ahead of the crack tip, appearing as an equilateral triangle with the crack tip as its base. This damage zone is known as the failure process zone, which is a key parameter in viscoelastic fracture mechanics under mode I type loading. When the local strain reaches a critical value, small voids are generated in the failure process zone. Because of the random nature of the microstructure, the first void is not restricted to the surface where the maximum normal strain occurs. Since the tendency of the filler particle to separate from the binder under a triaxial loading condition is high, it is expected that voids, or a damage zone, will also be generated in the specimen's interior. Consequently, there are a large number of strands, essentially made of the rubbery binder material, which separate the voids that form inside the failure process zone. Under this condition, the transverse constraint is minimized, approaching a state of plane stress condition. As the applied strain increases with time, material fracture occurs at the blunted end of the crack tip. This will always be the location of the maximum local strain. The failure of the material between the void and the crack tip causes the crack to grow into the failure process zone. This kind of crack growth mechanism continues until the main crack tip reaches the front of the failure process zone. When this occurs, the crack tip resharpens temporarily prior to accumulative of damage and blunting

The damage and crack growth mechanisms discussed above are the basic mechanisms observed in this material under both ambient and 6.9 MPa pressures. The effect of pressure is to suppress the damage and evolution processes and delay the onset of crack growth.

A plot of crack length versus time is shown in Fig. 6, in which the maximum applied load is also marked on the crack growth curve. It is interesting to point out that under confining pressure, the crack grows stably not only beyond the maximum applied stress but also near the break of the specimen. This phenomenon is significantly different from the crack growth behavior of the same material under ambient pressure. Under ambient pressure, crack growth becomes unstable when the applied load exceeds the maximum applied load.

The determination of the crack growth rate requires an analysis of discrete data relating the instantaneous time $t$ to the corre- 


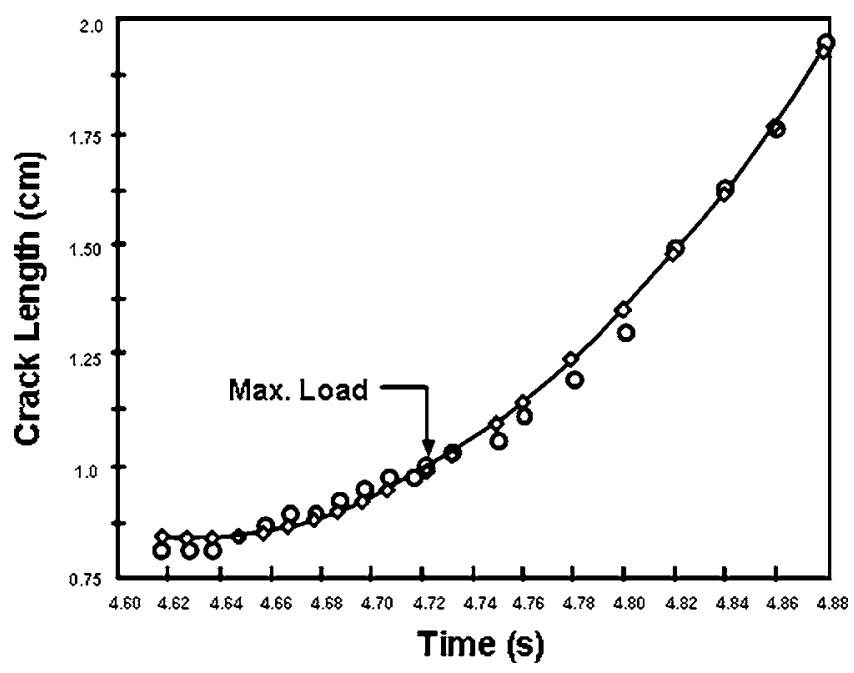

Fig. 6 Crack length versus time curve

sponding crack length $a$. Because of the inhomogeneous nature of the highly filled elastomer the measured data showed a considerable scatter. Therefore, it was anticipated that a smooth and steadily increasing relationship between the crack growth rate and time was difficult to obtain, and different methods of calculating $d a / d t$ calculation might result in different quantities. As mentioned earlier, the polynomial method was used to calculate the crack growth rate in this study. The smooth action introduced by the polynomial method results in a continuous smooth crack velocity curve. There is another method, known as the secant method [9], that can be used to calculate the crack growth rate. In the secant method, the crack growth rate was computed by calculating the slope of a straight line connecting two adjacent $a$ versus $t$ data points and assigning the average crack growth rate to the middle point between each pair of data points. Based on our experience, the secant method introduces a pronounced fluctuation in the crack growth rate $d a / d t$. In other words, the crack growth process consists of a slow-fast-slow phenomenon. As mentioned earlier, the damage process is a time-dependent process and requires finite time to develop a failure process zone at the crack tip. Thus, the crack growth process consists of blunt-growth-blunt and slow-fast-slow phenomena, which is highly nonlinear. The fluctuation in crack growth rate, $d a / d t$, is consistent with experimental observation. Based on experimental evidence, in general, the crack does not grow in a continuous and smooth manner. During the crack growth process, crack growth rate both accelerates and decelerates. Therefore, the secant method appears to provide the best emulation of the crack growth process and the actual crack growth rate. It is important and interesting to point out that, in general, the crack growth rate calculated by the secant method oscillate approximately around the smooth crack growth rate curve obtained by the polynomial method. Therefore, it is reasonable to expect that the crack growth velocity calculated by the polynomial method may represent the mean crack growth rates with the crack growth rate calculated by the secant method oscillating around it.

When the crack growth rate fluctuates, there will be no one-toone correspondence between the crack growth velocity and the stress intensity factor. This contradicts current crack growth theory for viscoelastic materials, which requires a unique relationship between the crack growth rate and the stress intensity factor. The lack of uniqueness between the crack growth rate and the stress intensity factor raises two questions: to what extent should the oscillation of the crack growth rate be taken into account and how to present and use these data. How these questions are answered depends on how the gathered data will be used. If one is interested in knowing the central tendency of the crack growth

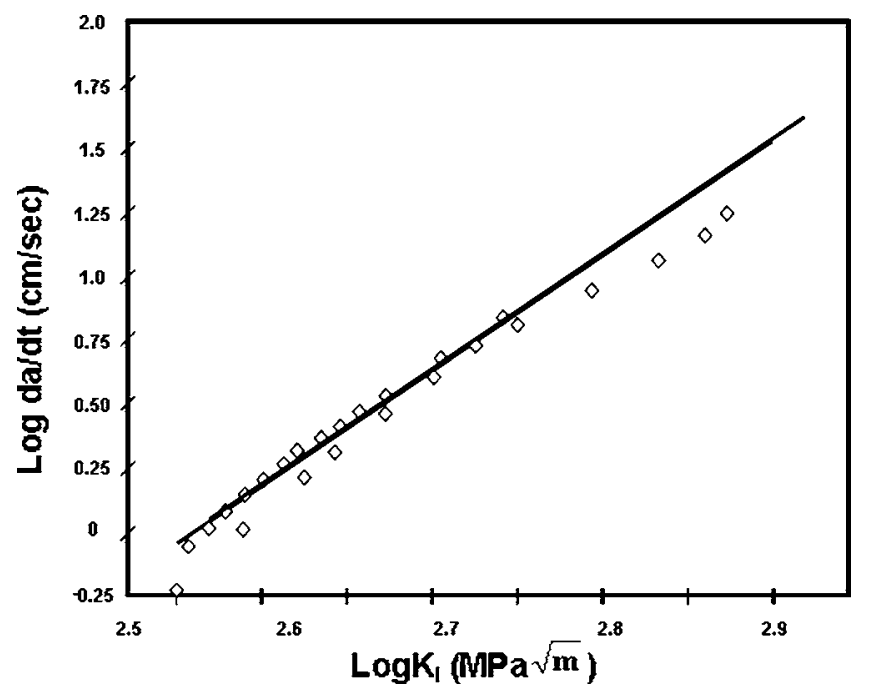

Fig. 7 Crack growth rate versus mode I stress intensity factor (pressure $=3.45 \mathrm{MPa}$ )

behavior or comparing the average crack growth behavior under different loading conditions, the fluctuation in the crack growth rate seems unimportant and it recommends using the polynomial method to calculate the crack growth rate. However, if one is interested in determining the upper-bound limit on the crack growth rate, the fluctuation in the crack growth rate should be considered in the data analysis and the secant method should be used to calculate the crack growth rate.

Typical plots of the crack growth rate $d a / d t$ versus the stress intensity factor $K_{\mathrm{I}}$ for different confining pressures are shown in Figs. 7 and 8. According to Figs. 7 and 8, power-law relationships appear to exist between the crack growth rate and the mode I stress intensity factor, which support the theories developed by Knauss [5] and Schapery [6] in their studies of crack growth behavior in linear viscoelastic materials. The effect of confining pressure on the crack growth behavior can be determined by comparing Fig. 7 to Fig. 8. It is noted that crack grows slower under a higher confining pressure, for reason discussed earlier.

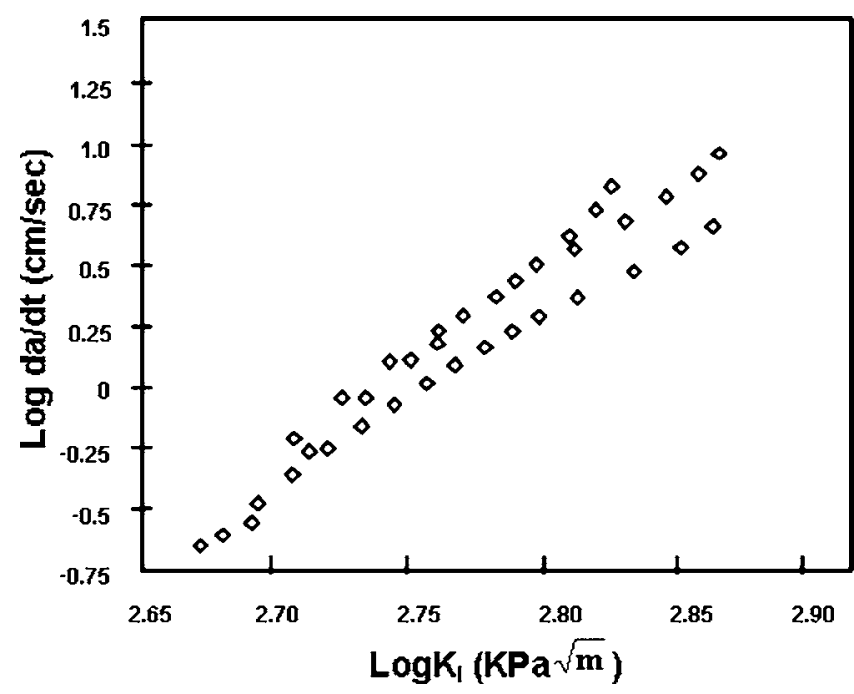

Fig. 8 Crack growth rate versus mode I stress intensity factor (pressure $=6.90 \mathrm{MPa}$ ) 


\section{Numerical Modeling}

Stress-strain response and crack initiation at room temperature in the highly filled elastomer was analyzed at different confining pressures using a modified version of a phenomenological damage model developed earlier [7] and accounting for time dependence of the homogenized material. Experimental observations, based on dilatation tests conducted under a different program, show that the volume dilatation $\Theta$ (damage parameter in RL model [7]) is independent of the loading rate and purely a function of applied strain. In other words, volume dilatation is dependent only on mechanical deformation (strain), and hence, damage is not a timedependent process. Taking these observations into account, the material time dependence is introduced through a simple Maxwell model for the undamaged material, and it is assumed that both the bulk and shear response of the material are governed by the same relaxation time $\tau$. The undamaged material's bulk $\left(\kappa_{0}\right)$ and shear $\left(\mu_{0}\right)$ moduli are given in terms of its rubbery moduli, $\kappa_{\infty}$ and $\mu_{\infty}$ and Prony coefficients $\kappa_{1}$ and $\mu_{1}$

$$
\kappa_{0}(t)=\kappa_{\infty}+\kappa_{1} \exp (-t / \tau) \text { and } \mu_{0}(t)=\mu_{\infty}+\mu_{1} \exp (-t / \tau)
$$

Stress components $\sigma_{i j}$ are evaluated using the convolution integral

$$
\sigma_{i j}(t)=\int_{0}^{t} \kappa_{0}\left(t-t^{\prime}\right) g(\Theta) \dot{\Theta} \delta_{i j} d t^{\prime}+\int_{0}^{t} 2 \mu_{0}\left(t-t^{\prime}\right) h(\Theta) \dot{\varepsilon}_{i j} d t^{\prime}
$$

where $g$ and $h$ are the damage parameters, which are purely functions of the volume dilatation.

The viscoelastic material parameters in Eq. (1) are obtained by fitting uniaxial stress-strain data at two different loading rates. The modified viscoelastic model has been implemented in a special purpose displacement based finite element code FEAP-SP. The damage parameters governing the degradation of the bulk and the shear moduli, $g$ and $h$, are determined using the uniaxial response and dilatation data as outlined in the original model [7]. The finite element method uses the Newton-Raphson iterative procedure for achieving force equilibrium and Newton's iterative method for enforcing plane stress condition. Constitutive update (stress, dilatation) is performed through integration of Eq. (2) for a finite time step. Time steps are chosen to preserve accuracy in the constitutive update and to ensure quadratic convergence of the residual energy norm.

Crack initiation is simulated in specimens with edge cracks subjected to prescribed displacement rates at the boundaries. Because of the inherent nature of the singular stress fields near the crack tip, the loading rate varies by at least two orders of magnitude from the loading boundary to the crack tip. The relaxation and damage parameters determined from the uniaxial stress-strain curves are used in the finite element calculations to update the local moduli and the damage parameter. Crack initiation is deter- mined by the attainment of critical dilatation at a critical distance from the crack tip and is used as the failure criterion [8]. The finite element model continues check at the end of every time step elements that have attained the specified critical dilatation.

The simulations of crack initiation were carried out in the material at the two different confining pressures, $3.45 \mathrm{MPa}$ and 6.9 MPa. Displacements were specified at the specimen boundaries normal to the crack to maintain a specified loading rate of $16.7 \mathrm{~cm} / \mathrm{cm} / \mathrm{min}$. The load increased nearly linearly with increasing displacement with the crack blunting monotonically. Upon reaching the critical dilatation in the crack tip vicinity, the crack was initiated. The predicted (measured) critical value for the stress intensity factor $\left(K_{\mathrm{Ic}}\right)$ for the onset of crack growth that were obtained for the two different confining pressures $3.45 \mathrm{MPa}$ and $6.9 \mathrm{MPa}$ are $0.220(0.230)$ and $0.238(0.243) \mathrm{MPa} \mathrm{m}^{1 / 2}$, respectively. The good correlation between the measured and the predicted $\mathrm{K}_{\mathrm{Ic}}$ validates the critical damage based simulations on the onset of crack growth.

\section{Conclusions}

In this study, the effects of confining pressure on the crack growth behavior were investigated. Experimental findings reveal that under confining pressure, cracks grow stably near the fracture of the specimen. They also reveal that, for a given mode I stress intensity factor, the crack growth rate decreases as the confining pressure is increased and a power-law relationship exists between the crack growth rate and the mode I stress intensity factor. In addition, a good correlation exists between the predicted and the measured $\mathrm{K}_{\mathrm{Ic}}$ for the onset of crack growth. Further investigations concerning the mechanics of the role of pressure on damage initiation and growth in highly filled elastomers is under way.

\section{References}

[1] Beckwith, S. W., and Wang, D. T., 1978, "Crack Propagation in Double-Base Propellants," AIAA Paper No. 78-170.

[2] Liu, C. T., 1990, "Crack Growth Behavior in a Composite Propellant With Strain Gradients-Part II,” J. Spacecr. Rockets, 27, pp. 647-659.

[3] Liu, C. T., 1990, "Crack Propagation in a Composite Solid Propellant," Proceedings of the Society of Experimental Mechanics, Spring Conference, SEM, Bethel, CT, pp. 614-620.

[4] Liu, C. T., and Smith, C. W., 1996, "Temperature and Rate Effects on Stable Crack Growth in a Particulate Composite Material," Exp. Mech., 36(3), pp. 290-295.

[5] Knauss, W. G., 1970, "Delayed Failure-The Griffith Problem for Linearly Viscoelastic Materials," Int. J. Fract. Mech., 6, pp. 7-20.

[6] Schapery, R. A., 1973, "On a Theory of Crack Growth in Viscoelastic Media," Report No. MM 2765-73-1, Texas A\&M University, College Station.

[7] Ravichandran, G., and Liu, C. T., 1995, "Modeling Constitutive Behavior of Particulate Composites Undergoing Damage," Int. J. Solids Struct., 32, pp. 979-990.

[8] Ravichandran, G., and Liu, C. T., 1998, "Crack-Tip Shielding in Particulate Composites Undergoing Damage," Eng. Fract. Mech., 59, pp. 713-723.

[9] Liu, C. T., 1990, "Critical Analysis of Crack Growth Data," J. Propul. Power, 6(5), pp. 519-524 